\title{
Effects of annatto-derived tocotrienol supplementation on osteoporosis induced by testosterone deficiency in rats
}

This article was published in the following Dove Press journal:

Clinical Interventions in Aging

5 August 2014

Number of times this article has been viewed

\author{
Kok-Yong Chin \\ Soelaiman Ima-Nirwana \\ Department of Pharmacology, Faculty \\ of Medicine, Universiti Kebangsaan \\ Malaysia, Kuala Lumpur, Malaysia
}

Background: Previous animal models have demonstrated that tocotrienol is a potential treatment for postmenopausal osteoporosis. This study evaluated the antiosteoporotic effects of annattoderived tocotrienol (AnTT) using a testosterone-deficient osteoporotic rat model.

Methods: Forty rats were divided randomly into baseline, sham, orchidectomized, AnTT, and testosterone groups. The baseline group was euthanized without undergoing any surgical treatment or intervention. The remaining groups underwent orchidectomy, with the exception of the sham group. AnTT $60 \mathrm{mg} / \mathrm{kg}$ /day was given orally to the AnTT group, while the testosterone group received testosterone enanthate $7 \mathrm{mg} / \mathrm{kg}$ per week intramuscularly for 8 weeks. Structural changes in trabecular bone at the proximal tibia were examined using microcomputed tomography. Structural and dynamic changes at the distal femur were examined using histomorphometric methods. Serum osteocalcin and C-terminal of type 1 collagen crosslinks were measured. Bone-related gene expression in the distal femur was examined.

Results: There were significant degenerative changes in structural indices in the orchidectomized group $(P<0.05)$, but no significant changes in dynamic indices, bone remodeling markers, or gene expression $(P>0.05)$ when compared with the sham group. The AnTT group showed significant improvement in structural indices at the femur $(P<0.05)$ and significantly increased expression of bone formation genes $(P<0.05)$. Testosterone was more effective than AnTT in preventing degeneration of bone structural indices in the femur and tibia $(P<0.05)$.

Conclusion: AnTT supplementation improves bone health in testosterone-deficient rats by enhancing bone formation. Its potential should be evaluated further by varying the dosage and treatment duration.

Keywords: bone remodeling, osteoporosis, testosterone, tocotrienol

\section{Introduction}

Although the prevalence of osteoporosis in men is lower than that in women, ${ }^{1}$ male osteoporosis is still regarded as a tremendous health problem. Men with osteoporosis suffer from higher mortality after a fracture when compared with their female counterparts. $^{2,3}$ Osteoporotic fractures also cause a substantial financial burden to society and decrease quality of life for patients, ${ }^{4,5}$ so should be prevented. The agerelated decrease in testosterone is a major risk factor for male osteoporosis. ${ }^{6,7}$ Several pharmacologic antiosteoporotic agents are available on the market, including bisphosphonates, teriparatide, and strontium ranelate, but are not free of side effects. ${ }^{8}$ Calcium supplementation with and without vitamin $\mathrm{D}$ is commonly prescribed, but has been associated with an increased risk of cardiovascular events. ${ }^{9}$ This has prompted a search for other agents with the potential to prevent osteoporosis.
Correspondence: Soelaiman Ima-Nirwana Department of Pharmacology, Faculty of Medicine, Universiti Kebangsaan Malaysia, Jalan Raja Muda Abdul Aziz, 50300 Kuala Lumpur, Malaysia

Tel +60340405514

Fax +60326938205

Email imasoel@ppukm.ukm.edu.my 
Tocotrienol is a subset of the vitamin E family, derived from plant oils, and has been shown to have antiosteoporotic effects. ${ }^{10,11}$ Previous studies in animal models of osteoporosis have demonstrated that mixtures of tocotrienols can prevent degenerative changes in trabecular bone induced by estrogen deficiency, ${ }^{12-14}$ free radicals, ${ }^{15}$ and nicotine. ${ }^{16}$ Tocotrienols have also been shown to preserve bone mineral density and calcium content in rats with dexamethasone-induced osteoporosis. ${ }^{17,18}$ These studies have used palm tocotrienols, which contain a substantial amount of alpha-tocopherol $(\alpha \mathrm{TF})$. Fujita et al have shown that $\alpha \mathrm{TF}$ promotes fusion of osteoclasts and increases bone resorption, ${ }^{19}$ which might interfere with the bone-protective effects of a tocotrienol mixture containing $\alpha \mathrm{TF}$. However, previous studies have not used molecular techniques, so the bone-protective mechanism of tocotrienol remains unclear.

Annatto-derived tocotrienol (AnTT) contains a high proportion of $\delta \mathrm{TT}$ and virtually no $\alpha \mathrm{TF},{ }^{20}$ so provides an opportunity to study the antiosteoporotic effects of tocotrienol without the interference of $\alpha \mathrm{TF}$. Abdul-Majeed et al found that osteoporotic changes due to estrogen deficiency were prevented in ovariectomized rats supplemented with AnTT $60 \mathrm{mg} / \mathrm{kg}$ for 8 weeks. ${ }^{21}$ We hypothesized that AnTT at this dose would be able to prevent male osteoporosis induced by testosterone deficiency.

In the current study, we evaluated the effects of AnTT on trabecular bone in orchidectomized rats with respect to changes in bone structural indices, remodeling, and gene expression. In addition to previously used bone histomorphometric methods, we used microcomputed tomography $(\mu \mathrm{CT})$ for more accurate three-dimensional estimation of bone structural parameters. Expression of genes related to bone formation and resorption was quantified to provide a mechanistic overview of how AnTT could prevent degenerative changes in bone. The information generated by this study could be used to determine whether AnTT acts as a preventive agent in male osteoporosis caused by testosterone deficiency. It also serves as a basis for use of AnTT in future clinical trials in humans.

\section{Materials and methods Materials}

The AnTT used in this study was a generous gift from American River Nutrition (Hadley, MA, USA). It was composed of approximately $90 \% \delta \mathrm{TT}$ and $10 \% \gamma \mathrm{TT}$, with no $\alpha \mathrm{TF}$. It was diluted in olive oil (Bartolini Emilio, Arrone Terni, Italy) and administered orally at a dose of $60 \mathrm{mg} / \mathrm{kg}$ body weight. Olive oil was used for dilution because it contains a low level of vitamin E (51 ppm). ${ }^{22}$ Testosterone enanthate (Jesalis Pharma, Jena, Germany) was diluted with peanut oil (Sime Darby, Subang Jaya, Malaysia) and administered intramuscularly at a dose of $7 \mathrm{mg} / \mathrm{kg}$ body weight. Peanut oil was used for dilution because the formulation used contains peanut oil.

\section{Treatment of animals}

Forty 3-month-old intact Sprague-Dawley rats (250-300 g) were purchased from the Laboratory Animal Research Unit, Universiti Kebangsaan Malaysia, Kuala Lumpur, Malaysia. They were acclimatized at the local vivarium for one week (room temperature, 12-hour light/dark cycle). The rats were housed singly in plastic cages with free access to tap water and standard rat chow (Gold Coin, Port Klang, Malaysia). They were stratified into five groups using random numbers generated by statistical software. Orchidectomy was performed in the orchidectomized, AnTT, and testosterone groups. A shamoperated group underwent similar surgical treatment but their testes were not removed. The baseline group was sacrificed without undergoing any surgical treatment or intervention. The AnTT group received AnTT daily (60 mg/kg body weight, oral gavage) for 8 weeks, while the other groups received an equal volume of the olive oil vehicle. The testosterone group received weekly testosterone enanthate injections ( $7 \mathrm{mg} / \mathrm{kg}$ body weight, intramuscularly) for 8 weeks while the other groups received an equal volume of the peanut oil vehicle. Treatment was initiated a week after orchidectomy. The weight of each rat was monitored weekly using a digital scale (Tanita Model 1,144, Tokyo, Japan) and recorded to the nearest $1 \mathrm{~g}$. At the end of the treatment period, the rats were euthanized using diethyl ether (HmbG Chemicals ${ }^{\circledR}$, Hamburg, Germany), after which their femoral and tibial bones were harvested. After the soft tissue was removed, the bones were either stored in $10 \%$ formalin solution for histomorphometric and $\mu \mathrm{CT}$ evaluation, or at $-70^{\circ} \mathrm{C}$ for study of gene expression. The study protocol was reviewed and approved by the animal ethics committee at Universiti Kebangsaan Malaysia (Code FP/FAR/2012/IMA/18-July/445-July-2012-April-2014).

\section{MicroCT analysis}

Trabecular parameters were investigated at the left proximal tibia using a $\mu$ CT 40 scanner (Scanco Medical, Brüttisellen, Switzerland). A sample was placed in a holder and scanned in an upright position. The volume of interest (200 slices) selected started from $1.5 \mathrm{~mm}$ distal to the lowest point of the proximal growth plate and extended towards the distal end of the tibia. Images were acquired at $70 \mathrm{kVp}$ and $114 \mu \mathrm{A}$ with high resolution. The integration time was $200 \mathrm{msec}$. 
Contouring was performed using a semiautomated method to select the trabecular region. Three-dimensional reconstruction and evaluation were performed using Scanco software (Scanco Medical) to measure the following bone parameters: trabecular bone volume, trabecular number, trabecular separation, trabecular thickness, structural model index, and connectivity density.

\section{Bone processing and histomorphometry}

Fluorochrome labeling was performed using calcein (SigmaAldrich, St Louis, MO, USA) $20 \mathrm{mg} / \mathrm{kg}$ body weight administered intraperitoneally on days 9 and 2 before the rats were euthanized. Undecalcified left rat femurs were embedded in polymethyl methacrylate (Sigma-Aldrich) and sectioned at a thickness of $9 \mu \mathrm{m}$ using a microtome (Leica RM2235, Nussloch, German). Sections to be used for evaluation of bone structure were stained using the Von Kossa method, while unstained sections were used for assessment of bone dynamics.

The region assessed was the secondary spongiosa in the metaphyseal region, which is $3-7 \mathrm{~mm}$ from the lowest point of the growth plate and $1 \mathrm{~mm}$ from the lateral cortex. Structural parameters were investigated by an automated method using an image analyzer (MediaCybernetics Image Pro-Plus, Rockville, MD, USA). Dynamic parameters were investigated using a fluorescence microscope (Nikon Eclipse 80i, Tokyo, Japan) and measured manually using a Weibel grid. The structural parameters measured included trabecular bone volume, thickness, number, and separation. Dynamic parameters measured included single-labeled surface, double-labeled surface, mineralizing rate, mineral apposition rate, and bone formation rate.

\section{Biochemical analysis}

Blood was collected from the rats prior to euthanasia via the retro-orbital sinus. Serum was extracted immediately after centrifuging the blood at 3,000 rpm for 10 minutes. The serum was stored at $-70^{\circ} \mathrm{C}$ until analysis. Osteocalcin and carboxy-terminal of type 1 collagen crosslinks were measured using an enzyme-linked immunosorbent assay kit (Immunodiagnostic Systems, Tyne and Wear, UK).

\section{Gene expression analysis}

A sample of trabecular bone was taken from the distal right femur and homogenized in buffer provided by the manufacturer using a high-speed homogenizer (Bead Ruptor 24, Omni, Kennesaw, GA, USA) and steel beads at $4^{\circ} \mathrm{C}$. The tissue lysate was incubated at $65^{\circ} \mathrm{C}$ and centrifuged to precipitate the debris.
mRNA expression was quantified using the QuantiGene Plex 2.0 $0^{\circledR}$ technique (Panomics/Affymetrix Inc, Santa Clara, CA, USA) according to the manufacturer's instructions. Oligonucleotide probe sets for the genes of interest were designed by the manufacturer. The tissue lysate was pipetted into a 96-well plate preloaded with capture reagent and a probe set. After incubation overnight at $54^{\circ} \mathrm{C}$, hybridization with the preamplifier, amplifier, and biotinylated label was carried out. Luminescence was measured using a Luminex ${ }^{\circledR}$ instrument (Bio-Rad, Hercules, CA, USA), and the mean fluorescence intensity specific for each gene (proportional to the mRNA captured by the bead) was generated. Expression of each gene was normalized to the expression of glyceraldehyde 3-phosphate dehydrogenase. The genes of interest are listed in Table 1.

\section{Statistical analysis}

Distribution of the data was assessed using the Shapiro-Wilk test and all data were found to be normally distributed. Oneway analysis of variance, with either Tukey's or Dunnett's $\mathrm{T} 3$ as the post hoc test, was used to detect significant differences in the variables of interest between the study groups. All data are presented as the mean and standard error of the mean. $P$-values $<0.05$ were considered to be statistically significant. The statistical analysis was performed using Statistical Package for Social Sciences version 16.0 software (SPSS Inc, Chicago, IL, USA).

\section{Results}

Rats in all the treatment groups gained weight throughout the study period. The percent increase in body weight was significantly less in the testosterone group than in the sham and AnTT groups $(P<0.05$, Figure 1A).

Orchidectomy resulted in decreased bone quality, as indicated by a significant reduction in trabecular bone volume, trabecular number, and connectivity density, and a significant increase in trabecular separation in the orchidectomized group when compared with the sham group using $\mu \mathrm{CT}$ at the proximal tibia $(P<0.05)$. Similar structural changes were observed at the distal femur, ie, the orchidectomized group showed significant reductions in trabecular bone volume, number, and thickness, and a significant increase in trabecular separation when compared with the sham group $(P<0.05)$. Treatment with testosterone was able to prevent these degenerative changes completely $(P<0.05)$, and treatment with tocotrienol was able to prevent adverse changes at the distal femur $(P<0.05)$. Structural indices at the proximal tibia showed a trend towards improvement in the AnTT group 
Table I Genes of interest in this study

\begin{tabular}{lll}
\hline Gene & NCBI accession number & Name \\
\hline Osteoblast-related & NM_0I3059 & \\
ALPL & Alkaline phosphatase \\
BGLAP & NM_0I34I4 & Osteocalcin or bone gamma-carboxyglutamate (gla) protein \\
COLIAI & Collagen, type I, alpha I \\
CTNNBI & NM_053357 & Catenin (cadherin associated protein), beta I \\
IBSP & NM_053470 & Integrin-binding sialoprotein \\
RUNX2 & NM_I8I374 & Runt-related transcription factor 2 \\
SP7 & NM_0I2656 & Sp7 transcription factor \\
SPARC & NM_0I2870 & Osteonectin or secreted protein, acidic, cysteine-rich \\
OPG/TNFRSFIIb & Osteoprotegerin/tumor necrosis factor receptor superfamily, member IIb \\
Osteoclast-related & NM_019144 & \\
ACP5 & NM_03I560 & Acid phosphatase 5, tartrate resistant \\
CTSK & NM_153720 & Cathepsin K \\
ITGB3 & IM_001058445 & Integrin, beta 3 \\
NFATCI & Nuclear factor of activated T-cells, cytoplasmic, calcineurin-dependent I \\
SPPI & NM_0I288I & Osteopontin or secreted phosphoprotein I or Bone sialoprotein I \\
SRC & NM_03I977 & Src v-src sarcoma (Schmidt-Ruppin A-2) viral oncogene homolog (avian) \\
RANKL/TNFSFII & NM_057I49 & Receptor activator of nuclear factor kappa-b/tumor necrosis factor \\
& & (ligand) superfamily, member II \\
Others & & \\
PPARG & NM_00II45366 & Peroxisome proliferator-activated receptor gamma, transcript variant 2 \\
\hline
\end{tabular}

\section{A Percentage change of body weight}

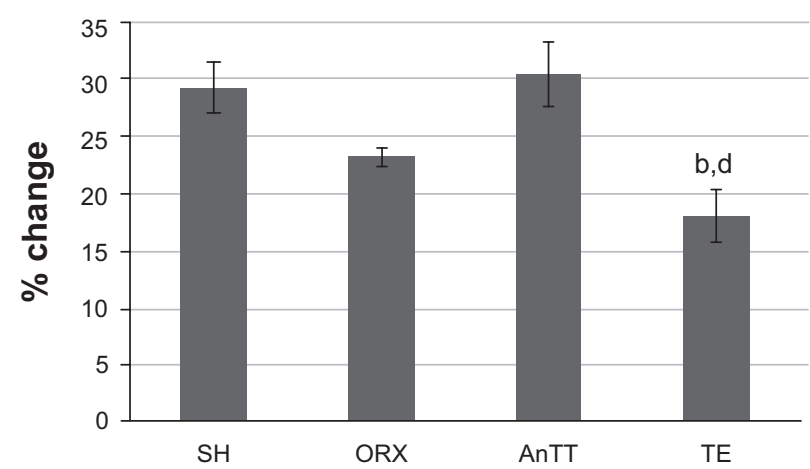

B Percentage change of CTX-1

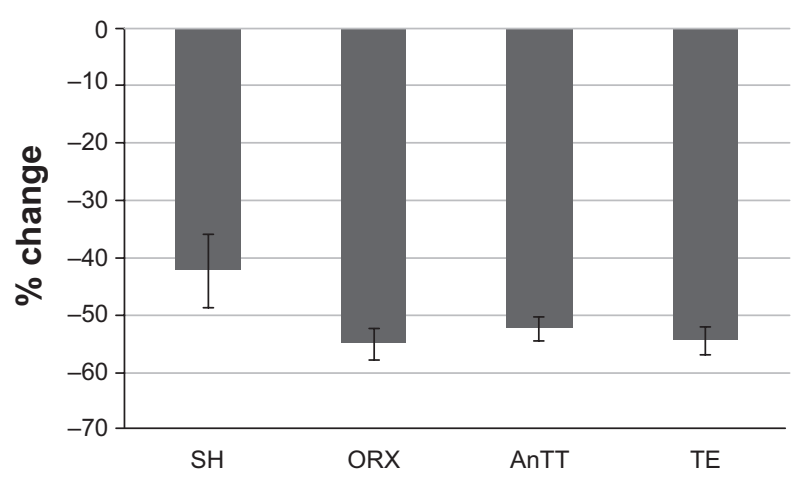

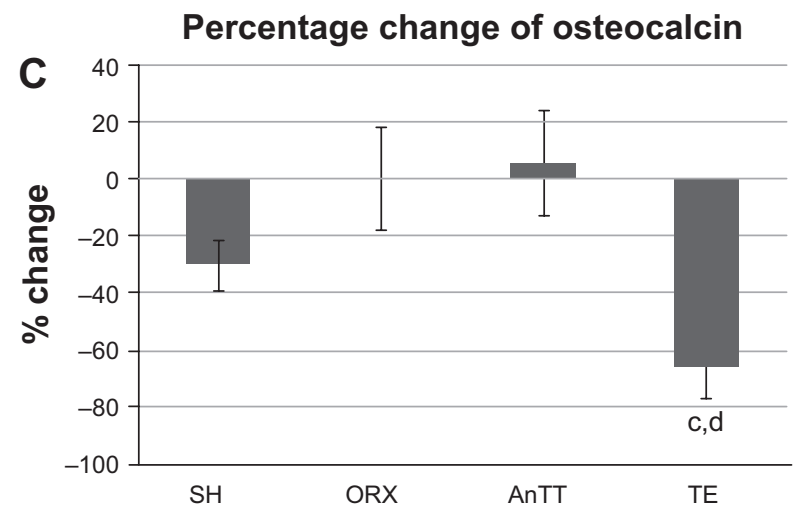

Figure I Percentage changes in body weight, serum terminal-C type I collagen crosslinks, and osteocalcin levels in rats. The bar charts show the percentage change in body weight (A), serum terminal-C type I collagen crosslinks (B), and osteocalcin levels (C) before and after treatment. The data are shown as the mean with the standard error of the mean.

Notes: 'Significant difference versus the sham group; 'significant difference versus the orchidectomized group; ${ }^{\mathrm{d}}$ significant difference versus the AnTT group. The statistical significance value is set at $P<0.05$.

Abbreviations: AnTT, annatto tocotrienol-supplemented group; BL, baseline group; CTX-I, terminal-C type I collagen crosslinks; ORX, orchidectomized group; SH, shamoperated group; TE, testosterone enanthate-supplemented group. 
when compared with the orchidectomized group, but only values for trabecular separation reached statistical significance $(P<0.05$; Figure 2 and Figure $3 \mathrm{~A}-\mathrm{D})$.

Dynamic histomorphometric variables (single-labeled surface, double-labeled surface, mineralizing rate, mineral apposition rate, and bone formation rate) were not significantly different between the orchidectomized and sham groups $(P>0.05)$. Treatment with testosterone suppressed bone remodeling, as evidenced by a significant reduction in double-labeled surface and a significant increase in
A Bone volume of proximal tibia

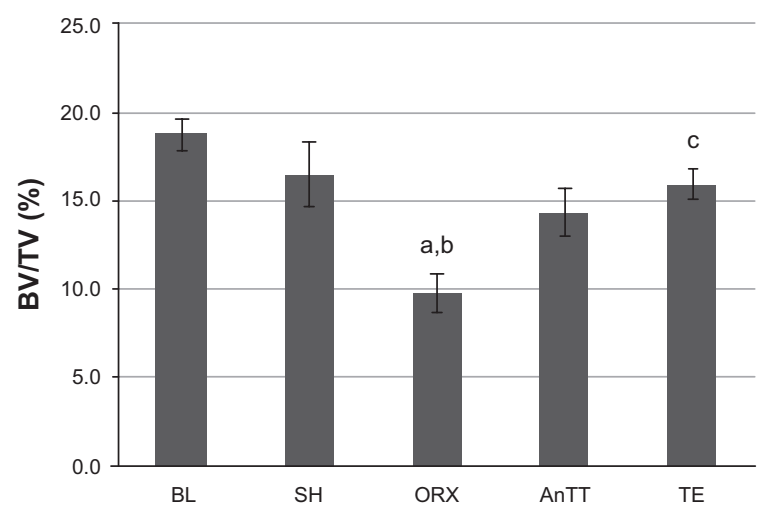

C

Trabecular thickness of proximal tibia

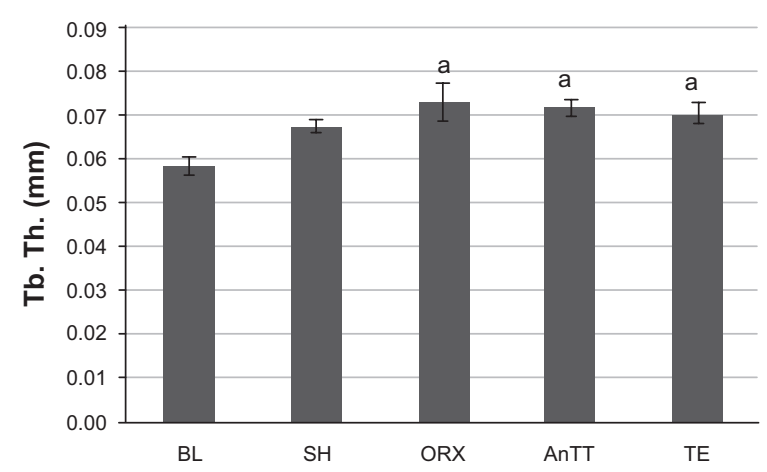

$\mathbf{E}$

Structural model index of proximal tibia

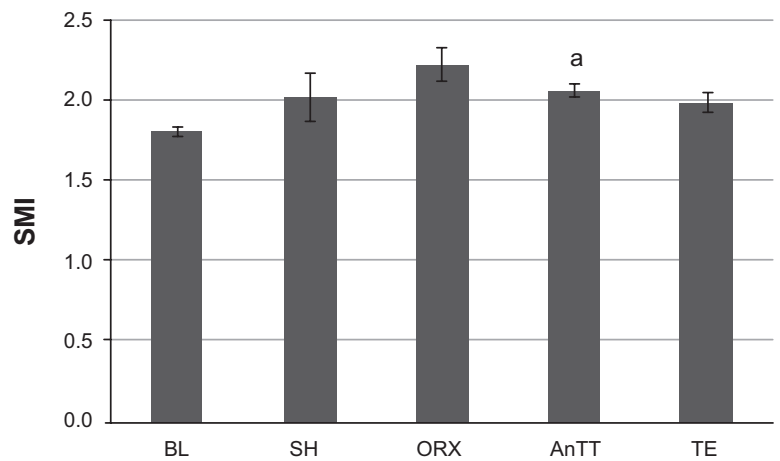

B Trabecular number of proximal tibia

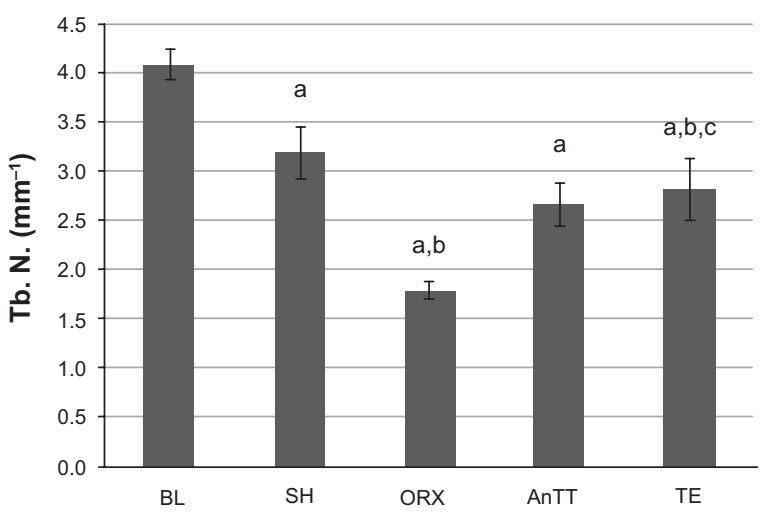

D

\section{Trabecular separation of proximal} tibia

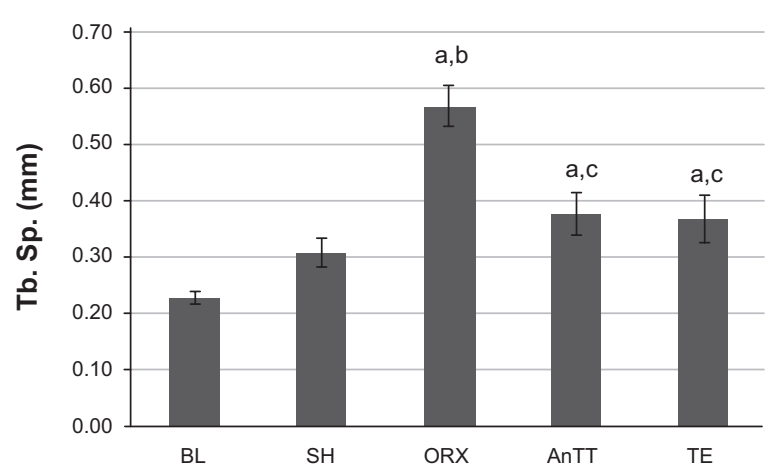

F Connectivity density of proximal tibia

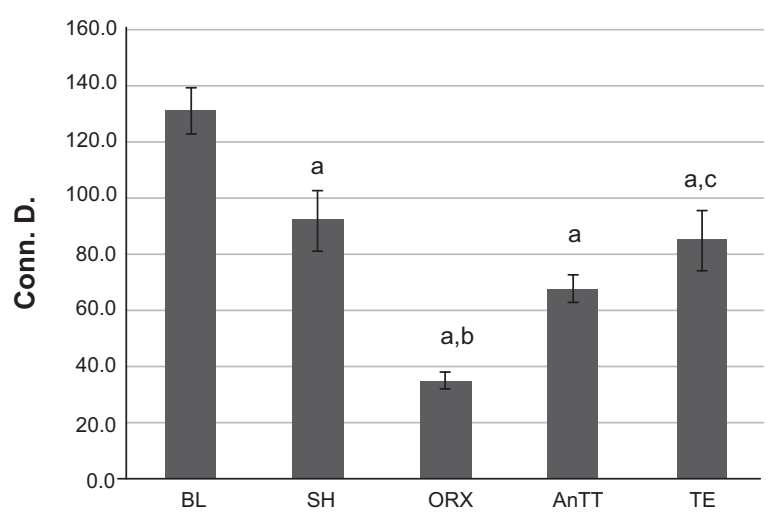

Figure 2 Bone structural indices in proximal tibia of rats evaluated using $\mu \mathrm{CT}$. The bar charts $(\mathbf{A}-\mathbf{F})$ show bone structural indices at the proximal tibia evaluated using $\mu \mathrm{CT}$. The data are shown as the mean and standard error of the mean.

Notes: a ${ }^{S}$ ignificant difference versus the baseline group; 'bignificant difference versus the sham group; 'significant difference versus the orchidectomized group. The statistical significance value is set at $P<0.05$.

Abbreviations: AnTT, annatto tocotrienol-supplemented group; BL, baseline group; ORX, orchidectomized group; SH, sham-operated group; TE, testosterone enanthatesupplemented group; SMI, Structural model index; Conn.D, connectivity density; BV/TV, bone volume over total volume; Tb. N., trabecular number; Tb. Th., trabecular thickness; Tb. Sp., trabecular separation. 

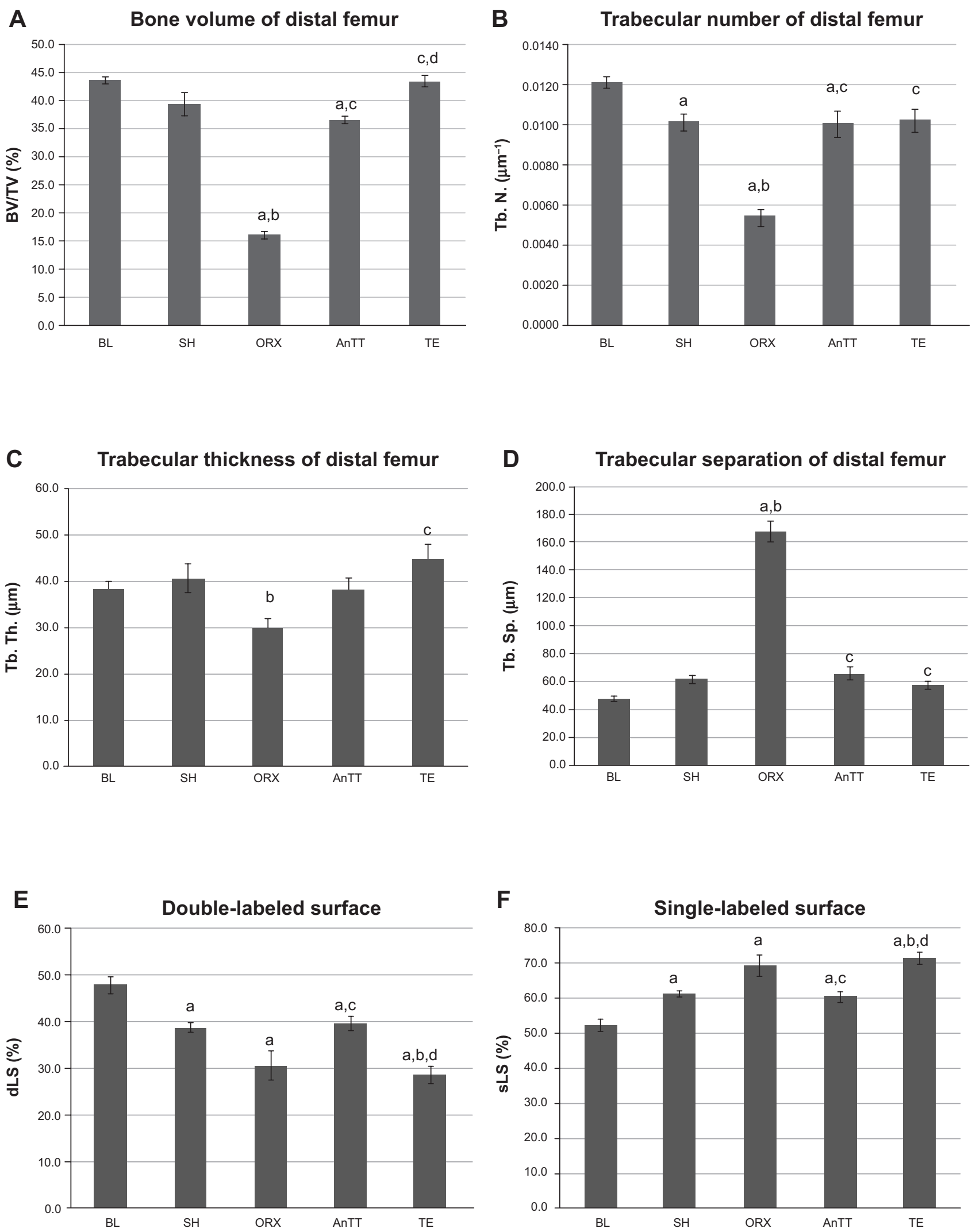

(Continued) 
G

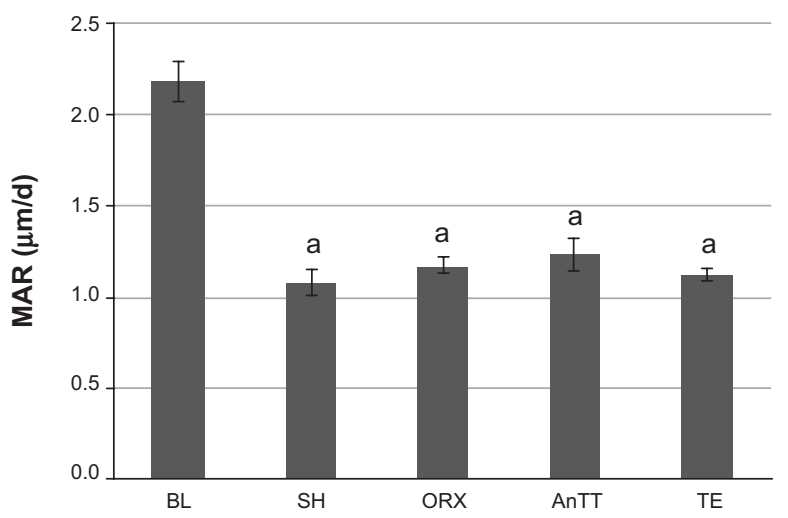

H

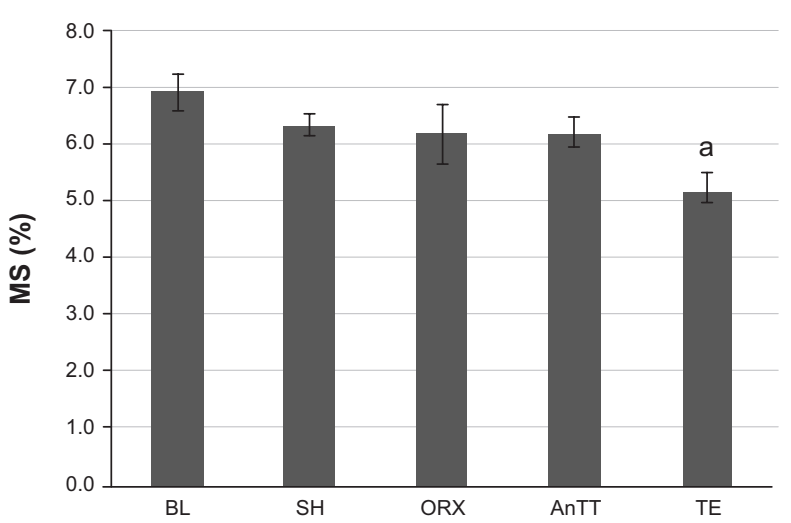

Bone formation rate

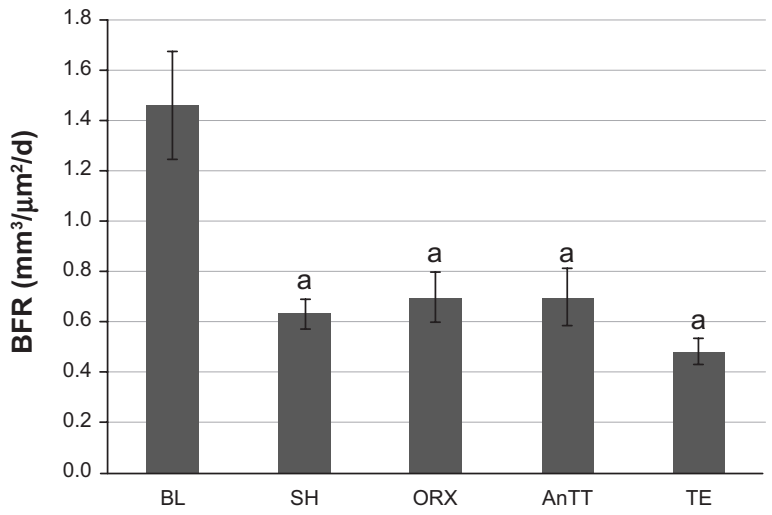

Figure 3 Bone structural and dynamic indices at the distal femur in rats evaluated using the bone histomorphometry technique. The bar charts show the bone structural (A-D) and dynamic (E-I) indices at the distal femur evaluated using the bone histomorphometry technique. The data are shown as the mean and standard error of the mean. Notes: aSignificant difference versus the baseline group; 'significant difference versus the sham group; 'significant difference versus the orchidectomized group; 'significant difference versus the AnTT group. The statistical significance value is set at $P<0.05$.

Abbreviations: AnTT, annatto tocotrienol-supplemented group; BL, baseline group; ORX, orchidectomized group; SH, sham-operated group; TE, testosterone enanthatesupplemented group; BV/TV, bone volume over total volume; Tb.N., trabecular number; Tb. Th., trabecular thickness; Tb. Sp., trabecular separation; dLS, double-labeled surface; sLS, single-labeled surface; MAR, mineral apposition rate; MS, mineralizing surface; BFR, bone formation rate.

single-labeled surface in the testosterone group as compared with the sham and AnTT groups $(P<0.05)$. Supplementation with tocotrienol increased bone remodeling, as indicated by a significant increase in double-labeled surface and a significant decrease in single-labeled surface $(P<0.05$; Figure 3E-I).

The difference between treatment with testosterone and treatment with AnTT with regard to the bone remodeling process was further supported by the bone turnover marker results. The orchidectomized group showed a marginally higher osteocalcin level when compared with the sham group, but the difference was not statistically significant $(P>0.05)$. Treatment with testosterone significantly suppressed osteocalcin levels in comparison with the orchidectomized group $(P<0.05)$. The tocotrienol-treated group showed higher osteocalcin levels than the orchidectomized group, although the difference was not statistically significant $(P>0.05)$. There was no difference in $\mathrm{C}$-terminal of type 1 collagen crosslink levels between the groups ( $P>0.05$; Figure 1B and C).
In general, gene expression was not significantly different between the sham and orchidectomized groups $(P>0.05)$. $R A N K L$ and $P P A R G$ gene expression was significantly lower in the testosterone group than in the orchidectomized group $(P<0.05)$. Treatment with tocotrienol significantly increased the expression of several bone formation genes, such as $A L P L$, when compared with the sham and orchidectomized groups $(P<0.05), C O L 1 \alpha 1$ when compared with the orchidectomized group $(P<0.05)$, and CTNNB1 when compared with the sham group $(P<0.05)$. Tocotrienol also significantly suppressed expression of $R A N K L$ and $P P A R G$ mRNA when compared with the orchidectomized group $(P<0.05)$. Expression of $R U N X-2$ and $S P A R C$ was also higher, albeit not significantly so, in the AnTT group when compared with the sham and orchidectomized groups $(P>0.05)$. There was no significant difference in expression of osteoclast-related genes between the groups with and without AnTT treatment $(P>0.05$; Figures 4 and 5$)$. 
A

ALPL expression

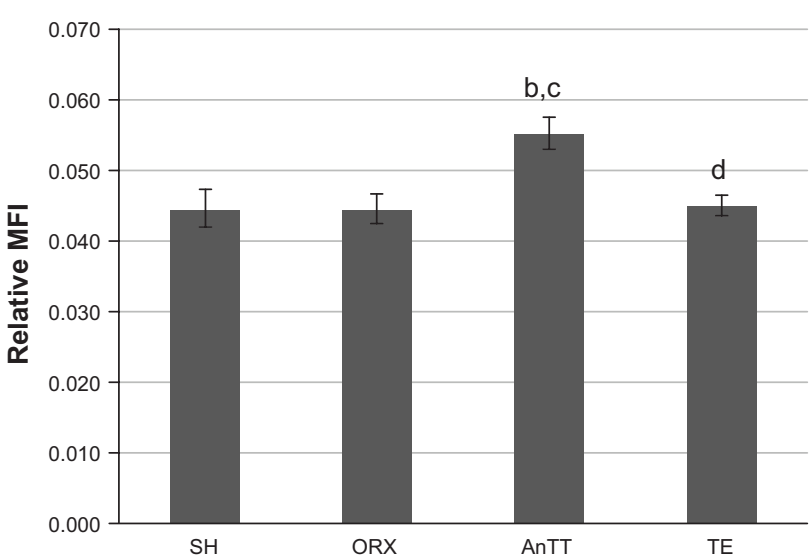

C



E

RUNX-2 expression

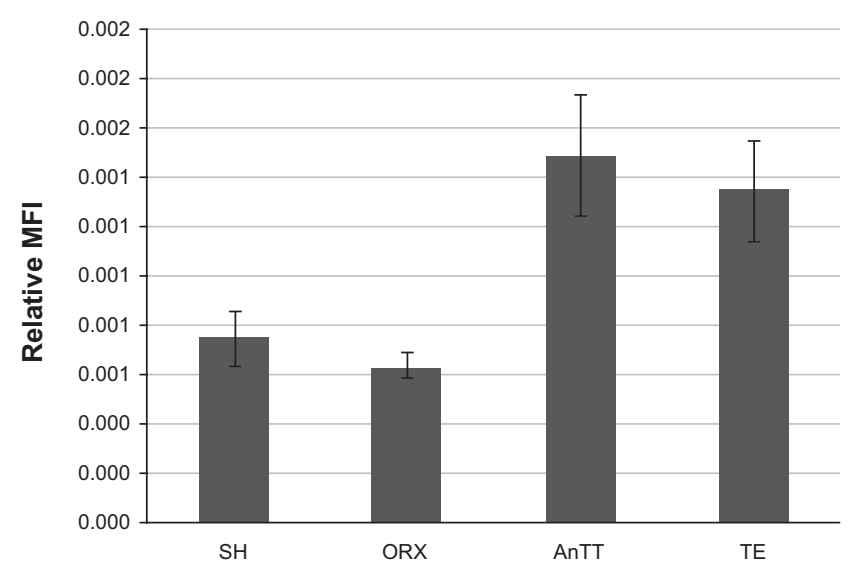

(Continued)
B

BGLAP expression

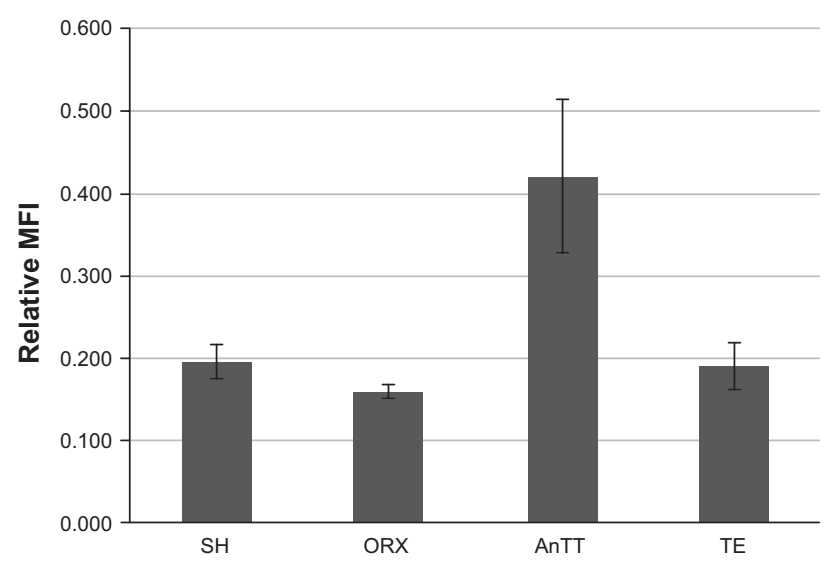

D

CTNNB expression

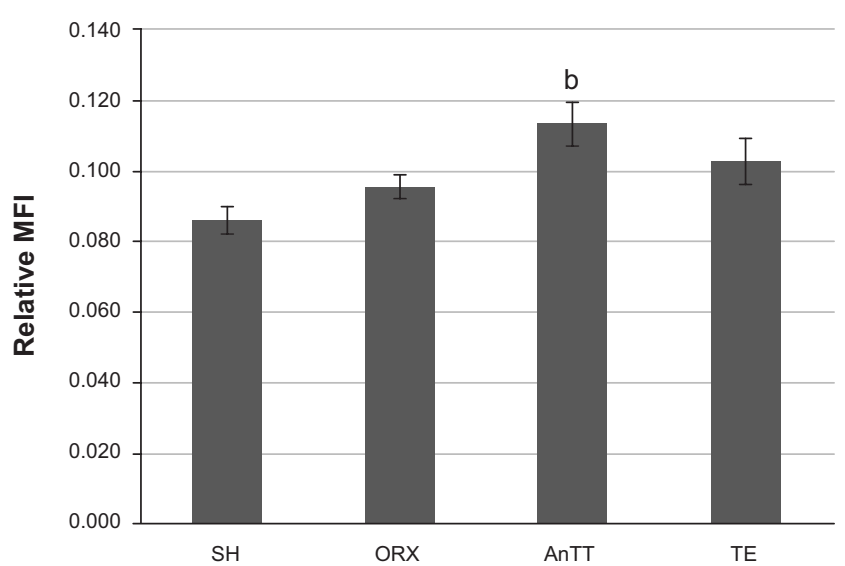

F

SP7 expression

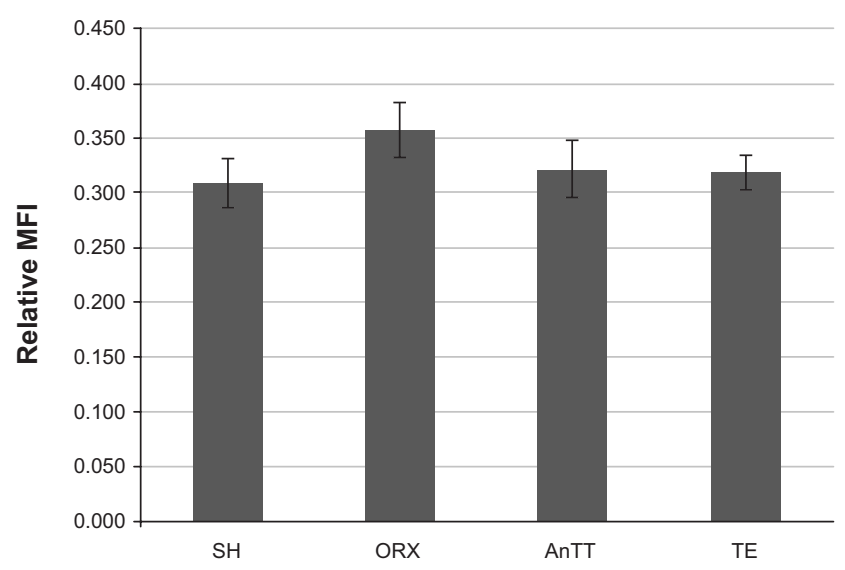


G



H

IBSP expression

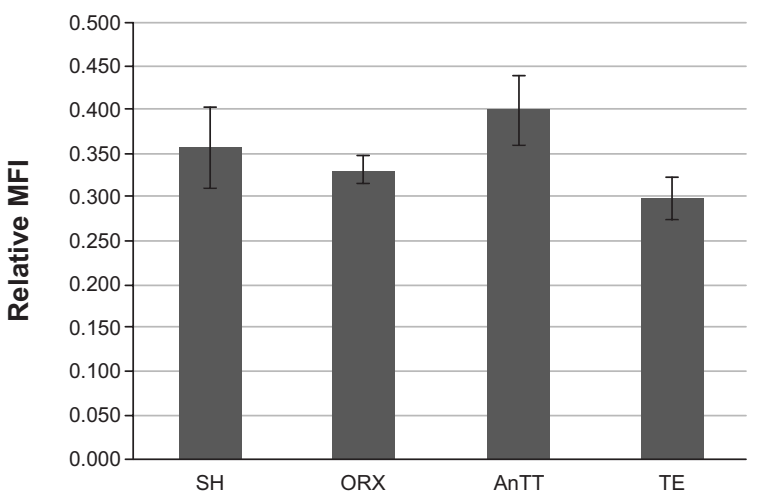

I

OPG expression

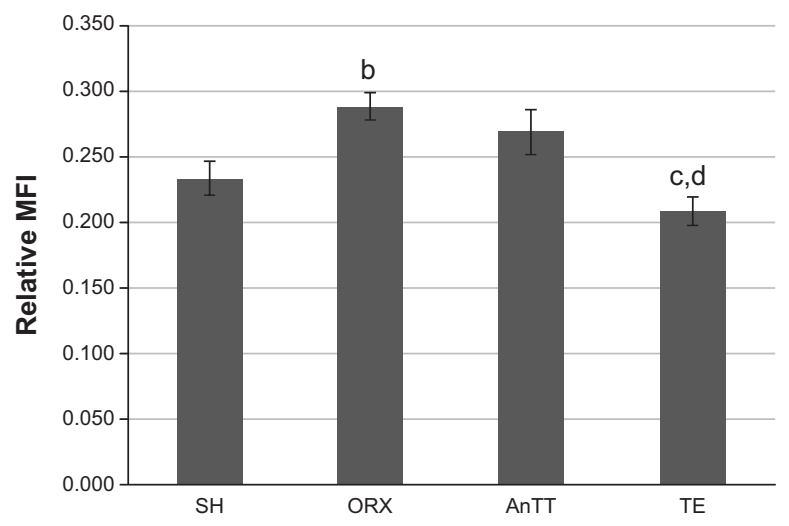

Figure 4 Relative expression of genes related to osteoblast differentiation/bone formation in rats. The bar charts (A-I) show the relative expression of genes related to osteoblast/bone formation. The data are shown as the mean with standard error of the mean.

Notes: bSignificant difference versus the sham group; 'significant difference versus the orchidectomized group; ${ }^{\mathrm{d}}$ significant difference versus the AnTT group. The statistical significance value is set at $P<0.05$.

Abbreviations: AnTT, annatto tocotrienol-supplemented group; BL, baseline group; ORX, orchidectomized group; SH, sham-operated group; TE, testosterone enanthatesupplemented group; MFI, mean fluorescence intensity.

\section{Discussion}

The present study indicates that osteoporosis induced by testosterone deficiency is characterized by a decrease in bone structural indices, such as trabecular bone volume, number, and thickness, as well as an increase in trabecular separation. These changes subsequently led to increased bone porosity, as evidenced by decreased connectivity density. There were no dramatic differences in indices of bone dynamics or bone remodeling markers between the orchidectomized and sham groups, probably because the increase in remodeling favorable to net bone loss was transient and had slowed down after 2 months. This also explains the lack of overt differences in gene expression profile between the orchidectomized and sham groups.

In the current study, the tocotrienol and testosterone interventions were found to preserve bone health in rats via two distinct mechanisms. Testosterone inhibited both bone formation (as evidenced by a significant decrease in double-labeled surface and serum osteocalcin level) and bone resorption (as evidenced by a significant decrease in RANKL mRNA expression), so bone remodeling was halted. Tocotrienol uncoupled bone remodeling by promoting bone formation (as evidenced by a significant increase in doublelabeled surface and expression of bone formation genes), with a negligible impact on bone resorption (as indicated by no significant changes in osteoclast-related gene expression). Differences between AnTT and testosterone with regard to the bone-protective mechanism have a significant clinical impact. We showed that testosterone was more effective than AnTT in preserving trabecular structure at the femur and tibia. However, inhibition of bone remodeling by testosterone could lead to accumulation of microfractures, thereby weakening the bone if not repaired via the normal remodeling process. On the other hand, AnTT activated bone formation but had negligible effects on bone resorption, resulting in a net gain of bone, which counteracted the loss of bone mass 
A

ACP5 expression

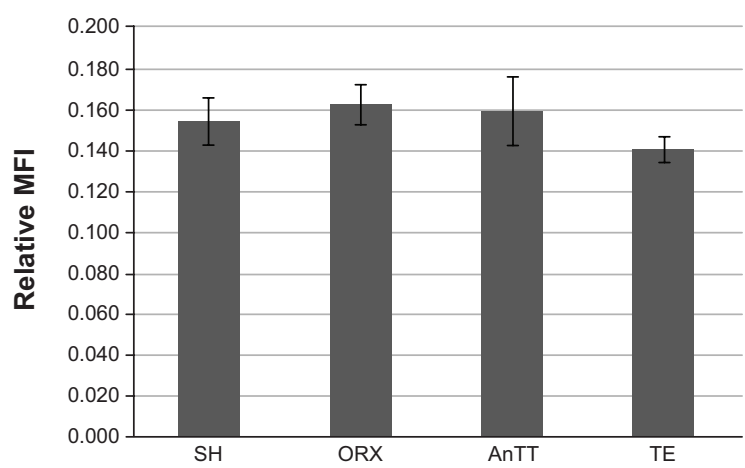

C

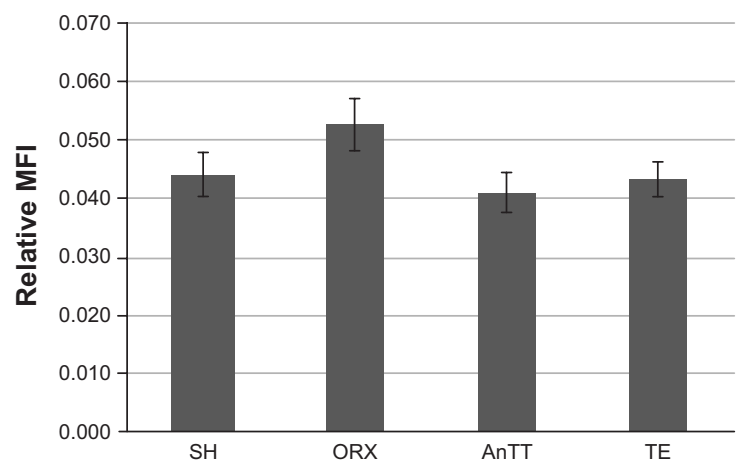

E



B

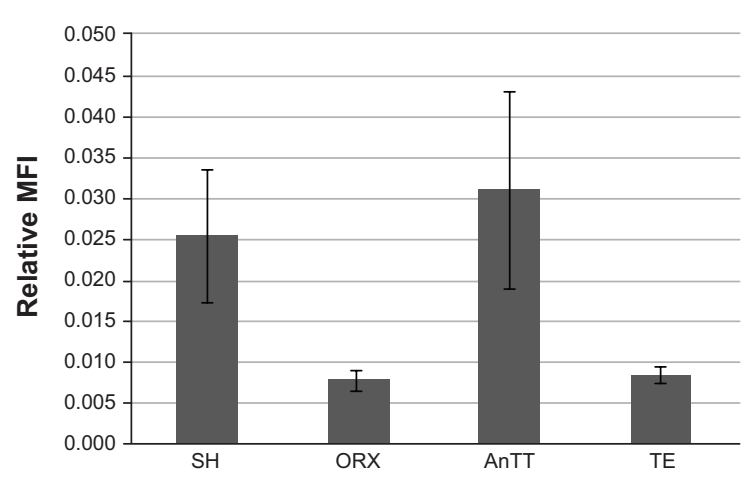

D



F

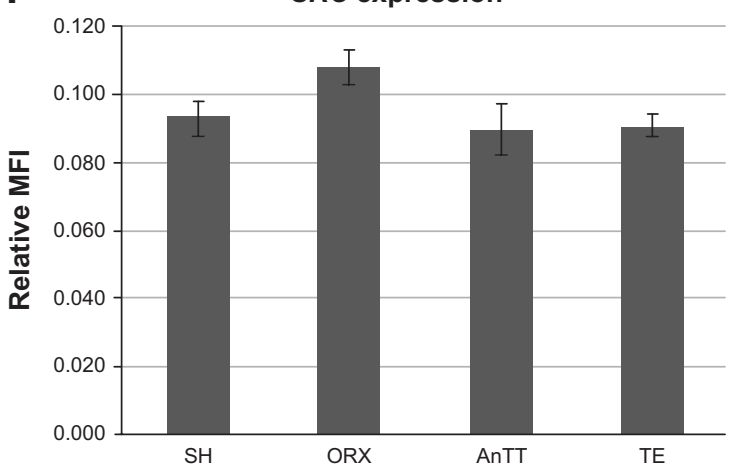

H

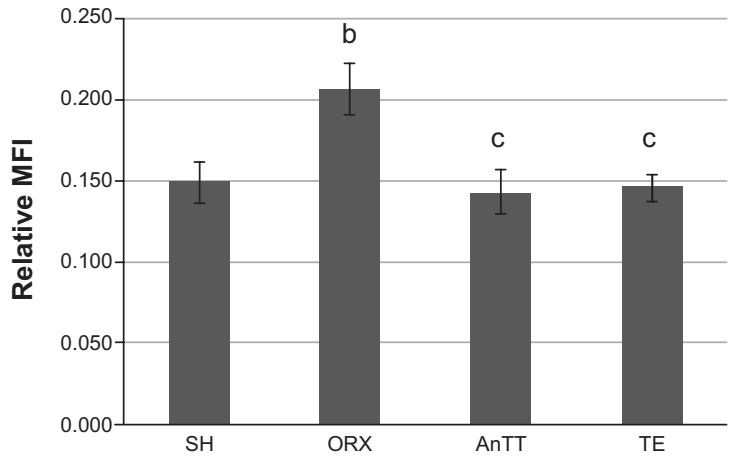

Figure $\mathbf{5}$ Relative expression of genes related to osteoclastogenesis/osteoclast function/bone resorption and PPARG in rats. The bar charts (A-H) show the relative expression of genes related to osteoclastogenesis/osteoclast function/bone resorption and PPARG. The data are shown as the mean and the standard error of the mean.

Notes: 'Significant differences versus the sham group; 'significant differences versus the orchidectomized group; 'significant difference versus the AnTT group. The statistical significance value is set at $P<0.05$.

Abbreviations: AnTT, annatto tocotrienol-supplemented group; BL, baseline group; ORX, orchidectomized group; SH, sham-operated group; TE, testosterone enanthatesupplemented group; MFI, mean fluorescence intensity. 
due to testosterone deficiency. Since bone remodeling was not suppressed, AnTT would allow repair of microfractures. However, examination of microfractures was outside the scope of the present study.

The use of a $\delta$ TT-predominant AnTT mixture without any $\alpha \mathrm{TF}$ is a novel approach in the prevention of male osteoporosis, so no comparable studies are available. A previous study by Abdul-Majeed et al showed that supplementing ovariectomized female rats with AnTT $60 \mathrm{mg} / \mathrm{kg}$ for 8 weeks prevented adverse changes in femoral trabecular bone due to estrogen deficiency, as evidenced by improvements in cellular and static bone parameters. ${ }^{21}$ Bone formation was increased and bone resorption was decreased in the AnTT-treated group in that study, ${ }^{21}$ whereas only bone formation was increased in the present study. This discrepancy might be due to sex differences in the response of bone to treatment with AnTT. Many studies had been performed previously using $\gamma \mathrm{TT}$ predominant palm-derived tocotrienol mixtures containing $20 \%-30 \% \alpha \mathrm{TF}$ to investigate the antiosteoporotic effects of tocotrienol. ${ }^{10}$ The majority of these studies found that palmderived tocotrienols at $60 \mathrm{mg} / \mathrm{kg}$ body weight were effective in preventing degenerative changes in structural, dynamic, cellular and static indices of bone in osteoporosis induced by estrogen deficiency, ${ }^{12-14}$ nicotine, ${ }^{16}$ and free radicals. ${ }^{15}$ In a study using normal rats, isomers of tocotrienol were found to have anabolic effects on bone as evaluated by biomechanical strength testing and histomorphometric evaluation. ${ }^{23}$ Hence, the current study showed that AnTT also exerted anabolic effects on bone in male rats with testosterone deficiency.

Very few studies have explored the bone-protective mechanism of tocotrienols, and the antiosteoclastogenic effects of tocotrienols were given more emphasis in the available in vitro studies. In vitro studies by Brooks et $\mathrm{al}^{24}$ and Ha et $\mathrm{a}^{25}$ demonstrated that tocotrienol isomers were able to decrease the formation and differentiation of osteoclasts from human CD14+ cells and mouse bone marrow macrophages and the resorption activity of osteoclasts. Ha et al further demonstrated that the antiosteoclastogenic effects of $\alpha \mathrm{TT}$ could be attributed to disruption of RANKL signaling. ${ }^{25}$ These observations were supported by several in vivo studies, in which eroded surface and osteoclast surface of trabecular bone were reduced in tocotrienol-supplemented female animals. ${ }^{21}$ The present research shows that AnTT supplementation significantly increases the expression of genes related to bone formation ( $A L P L, C O L 1 \alpha 1$, and CTNNB1) but does not have a significant influence on expression of genes related to osteoclasts. Although expression of RANKL was decreased in the AnTT group, expression of other genes related to osteoclast function ( $A C P 5, C T S K$, and $I T G B 3$ ) and signaling (NFATC1 and $S R C$ ) was not affected. Further, treatment with AnTT decreased expression of PPARG, which is responsible for differentiation of adipocytes, indicating that bone marrow mesenchymal cells were more committed to differentiate into osteoblasts (indicated by marginally higher but not statistically significant $R U N X$ expression in the AnTT group) instead of adipocytes. In view of previous evidence regarding the antiosteoclastogenic effects of tocotrienols, we postulate that the dose used in the current study was sufficient to activate genes for bone formation but not those for bone resorption. It is also possible that the effect of the AnTT mixture on bone remodeling was different from that of single tocotrienol isomers and palm-derived tocotrienol mixtures, whereby the AnTT mixture exerted predominantly anabolic but not antiresorptive effects in bone.

There are several advantages to using an $\alpha$ TF-free tocotrienol mixture. A study by Fujita et al showed that $\alpha$ TF stimulated osteoclast fusion and increased bone resorption activity, but other vitamin $\mathrm{E}$ isomers did not. ${ }^{19}$ Alpha-TF was also shown to compete with other vitamin $\mathrm{E}$ isomers to bind with $\alpha \mathrm{TF}$ transport protein, which secretes vitamin $\mathrm{E}$ isomers into the circulation, ${ }^{26}$ thus reducing the level of tocotrienol isomers beneficial to bone health. This was supported by intervention studies, in which $\alpha$ TF supplementation decreased circulating levels of other tocopherols and tocotrienols. ${ }^{27,28}$ These undesirable characteristics of $\alpha \mathrm{TF}$ might interfere with the bone-protective effects of tocotrienols and subsequently reduce their efficacy. ${ }^{29}$

Several limitations need to be considered in interpreting the results of the present study. The affinity of $\alpha \mathrm{TF}$ transport protein for $\delta \mathrm{TT}$ and $\gamma \mathrm{TT}$ was very low. ${ }^{26} \mathrm{We}$ did not determine the amount of tocotrienol that reached the circulation or bone. However, previous animal studies have shown that supplementation with tocotrienol $60 \mathrm{mg} / \mathrm{kg}$ improves bone quality. ${ }^{21}$ Trabecular bone was studied because it is metabolically active and provides a large surface to volume ratio for interaction between soluble factors and bone. ${ }^{30}$ Thus, trabecular bone would show a more rapid response than cortical bone when studying compounds that could affect bone. The antiosteoporotic effects of AnTT could be further explored by varying the dose and treatment duration. The dosage and treatment period in the current study were based on those used in an earlier ovariectomized osteoporosis model. ${ }^{21}$

This study is novel because the effects of an AnTT mixture with a unique composition of $90 \% \delta$ TT and $10 \% \gamma \mathrm{TT}$ have never been examined before in osteoporosis induced by testosterone deficiency. Previous studies have used 
palm-derived tocotrienol mixtures and focused on estrogeninduced osteoporosis and other models. The absence of $\alpha \mathrm{TF}$ in the mixture allows the effects of tocotrienols to be studied without undesirable interference. This study characterized the effects of AnTT on gene expression in bone, which has not been done by previous researchers. This allowed a better understanding of the mechanisms underlying the bone-protective action of AnTT. A gene expression study was performed using a multiplex platform that reduced errors due to sample processing, dilution, and amplification. The multiplex platform has been reported to have a lower coefficient of variation when compared with the traditional reverse transcriptase polymerase chain reaction. ${ }^{31}$

\section{Conclusion}

AnTT $60 \mathrm{mg} / \mathrm{kg}$ showed promising antiosteoporotic effects in rats with osteoporosis induced by testosterone deficiency. These antiosteoporotic effects are attributed to an increase in bone formation activity brought about by increased expression of genes related to bone formation. AnTT protects bone via a different mechanism when compared with testosterone replacement, which inhibits the bone modeling process. Further research of AnTT should be conducted by varying the dose and treatment duration, when examining the antiosteoporotic effects of AnTT in male osteoporosis induced by testosterone deficiency.

\section{Acknowledgments}

We thank the Universiti Kebangsaan Malaysia for funding this study via a grant from Impak Perdana (DIP-2012-07). We also thank Dr Lim Fei Tieng from Hi-Tech Instruments $\mathrm{Sdn}$. Bhd. for her assistance with the $\mu \mathrm{CT}$ measurements and Dr Thuan Bui from i-DNA Biotechnology Sdn. Bhd. for assistance in using the QuantiGene 2.0. We are also grateful for the assistance provided by the technicians in the Department of Pharmacology, Universiti Kebangsaan Malaysia.

\section{Disclosure}

The authors report no conflicts of interest in this work.

\section{References}

1. Johnell O, Kanis JA. An estimate of the worldwide prevalence and disability associated with osteoporotic fractures. Osteoporos Int. 2006; 17(12):1726-1733.

2. Morin S, Lix LM, Azimaee M, Metge C, Caetano P, Leslie WD. Mortality rates after incident non-traumatic fractures in older men and women. Osteoporos Int. 2011;22(9):2439-2448.

3. Kannegaard PN, van der Mark S, Eiken P, Abrahamsen B. Excess mortality in men compared with women following a hip fracture. National analysis of comedications, comorbidity and survival. Age Ageing. 2010;39(2):203-209.
4. Papaioannou A, Kennedy CC, Ioannidis G, et al. The impact of incident fractures on health-related quality of life: 5 years of data from the Canadian Multicentre Osteoporosis Study. Osteoporos Int. 2009; 20(5):703-714.

5. Burge R, Dawson-Hughes B, Solomon DH, Wong JB, King A, Tosteson A. Incidence and economic burden of osteoporosis-related fractures in the United States, 2005-2025. J Bone Miner Res. 2007;22(3):465-475.

6. Chin KY, Ima-Nirwana S. Sex steroids and bone health status in men. Int J Endocrinol. 2012;2012:208719.

7. Chin KY, Soelaiman IN, Mohamed IN, Ngah WZ. Serum testosterone, sex hormone-binding globulin and total calcium levels predict the calcaneal speed of sound in men. Clinics (Sao Paulo). 2012;67(8):911-916.

8. Varenna M, Bertoldo F, Monaco MD, Giusti A, Martini G, Rossini M. Safety profile of drugs used in the treatment of osteoporosis: a systematical review of the literature. Reumatismo. 2013;65(4):143-166.

9. Bolland MJ, Grey A, Avenell A, Gamble GD, Reid IR. Calcium supplements with or without vitamin D and risk of cardiovascular events: reanalysis of the Women's Health Initiative limited access dataset and meta-analysis. BMJ. 2011;342:d2040.

10. Chin K-Y, Ima-Nirwana S. Vitamin E as an antiosteoporotic agent via receptor activator of nuclear factor kappa-B ligand signaling disruption: current evidence and other potential research areas. Evid Based Complement Alternat Med. 2012;2012:747020.

11. Chin K-Y, Mo H, Soelaiman I-N. A review of the possible mechanisms of action of tocotrienol - a potential antiosteoporotic agent. Curr Drug Targets. 2013;14(13):1533-1541.

12. Aktifanus AT, Shuid AN, Rashid NHA, et al. Comparison of the effects of tocotrienol and estrogen on the bone markers and dynamic changes in postmenopausal osteoporosis rat model. Asian Journal of Animal and Veterinary Advances. 2012;7(3):225-234.

13. Muhammad N, Luke DA, Shuid AN, Mohamed N, Soelaiman IN. Two different isomers of vitamin e prevent bone loss in postmenopausal osteoporosis rat model. Evid Based Complement Alternat Med. 2012;2012:161527.

14. Soelaiman IN, Ming W, Abu Bakar R, et al. Palm tocotrienol supplementation enhanced bone formation in oestrogen-deficient rats. Int J Endocrinol. 2012;2012:7.

15. Ahmad NS, Khalid BA, Luke DA, Ima Nirwana S. Tocotrienol offers better protection than tocopherol from free radical-induced damage of rat bone. Clin Exp Pharmacol Physiol. 2005;32(9):761-770.

16. Hermizi H, Faizah O, Ima-Nirwana S, Ahmad Nazrun S, Norazlina M. Beneficial effects of tocotrienol and tocopherol on bone histomorphometric parameters in Sprague-Dawley male rats after nicotine cessation. Calcif Tissue Int. 2009;84(1):65-74.

17. Ima Nirwana $S$, Suhaniza S. Effects of tocopherols and tocotrienols on body composition and bone calcium content in adrenalectomized rats replaced with dexamethasone. $J$ Med Food. 2004;7(1):45-51.

18. Ima-Nirwana S, Fakhrurazi H. Palm vitamin E protects bone against dexamethasone-induced osteoporosis in male rats. Med J Malaysia. 2002;57(2):133-141.

19. Fujita K, Iwasaki M, Ochi H, et al. Vitamin E decreases bone mass by stimulating osteoclast fusion. Nat Med. 2012;18(4):589-594.

20. Frega N, Mozzon M, Bocci F. Identification and estimation of tocotrienols in the annatto lipid fraction by gas chromatography-mass spectrometry. J Am Oil Chem Soc. 1998;75(12):1723-1727.

21. Abdul-Majeed S, Mohamed N, Soelaiman I-N. Effects of tocotrienol and lovastatin combination on osteoblast and osteoclast activity in estrogen-deficient osteoporosis. Evid Based Complement Alternat Med. 2012;2012:960742.

22. Pierpaoli E, Viola V, Barucca A, Orlando F, Galli F, Provinciali M. Effect of annatto-tocotrienols supplementation on the development of mammary tumors in HER-2/neu transgenic mice. Carcinogenesis. 2013; 34(6):1352-1360.

23. Mehat M, Shuid A, Mohamed N, Muhammad N, Soelaiman I. Beneficial effects of vitamin $\mathrm{E}$ isomer supplementation on static and dynamic bone histomorphometry parameters in normal male rats. J Bone Miner Metab. 2010;28(5):503-509. 
24. Brooks R, Kalia P, Ireland D, Beeton C, Rushton N. Direct inhibition of osteoclast formation and activity by the vitamin $\mathrm{E}$ isomer gammatocotrienol. Int J Vitam Nutr Res. 2011;81(6):358-367.

25. Ha H, Lee J-H, Kim H-N, Lee ZH. $\alpha$-Tocotrienol inhibits osteoclastic bone resorption by suppressing RANKL expression and signaling and bone resorbing activity. Biochem Biophys Res Commun. 2011; 406(4):546-551.

26. Hosomi A, Arita M, Sato Y, et al. Affinity for $\alpha$-tocopherol transfer protein as a determinant of the biological activities of vitamin $E$ analogs FEBS Lett. 1997;409(1):105-108.

27. Huang H-Y, Appel LJ. Supplementation of diets with $\alpha$-tocopherol reduces serum concentrations of $\gamma$ - and $\delta$-tocopherol in humans. $J$ Nutr. 2003;133(10):3137-3140.
28. Ikeda S, Tohyama T, Yoshimura H, Hamamura K, Abe K, Yamashita K. Dietary $\alpha$-tocopherol decreases $\alpha$-tocotrienol but not $\gamma$-tocotrienol concentration in rats. $J$ Nutr. 2003;133(2):428-434.

29. Chin KY, Ima-Nirwana S. The effects of alpha-tocopherol on bone: a double-edged sword? Nutrients. 2014;6(4):1424-1441.

30. Jacobs CR. The mechanobiology of cancellous bone structural adaptation. J Rehabil Res Dev. 2000;37(2):209-216.

31. Canales RD, Luo Y, Willey JC, et al. Evaluation of DNA microarray results with quantitative gene expression platforms. Nat Biotechnol. 2006;24(9):1115-1122.
Clinical Interventions in Aging

\section{Publish your work in this journal}

Clinical Interventions in Aging is an international, peer-reviewed journal focusing on evidence-based reports on the value or lack thereof of treatments intended to prevent or delay the onset of maladaptive correlates of aging in human beings. This journal is indexed on PubMed Central, MedLine,

\section{Dovepress}

CAS, Scopus and the Elsevier Bibliographic databases. The manuscript management system is completely online and includes a very quick and fair peer-review system, which is all easy to use. Visit http://www.dovepress. $\mathrm{com} /$ testimonials.php to read real quotes from published authors. 